JOURNAL OF

FUNCTION SPACES AND APPLICATIONS

Volume 7, Number 2 (2009), 153-166 (c) 2009, Scientific Horizon

http://www.jfsa.net

\title{
Atomic decompositions of Lorentz martingale spaces and applications
}

\author{
Jiao Yong, Peng Lihua and Liu Peide
}

(Communicated by Maria Carro)

2000 Mathematics Subject Classification. Primary 60G42; Secondary $60 \mathrm{G} 46$.

Keywords and phrases. Atomic decompositions, martingale inequalities, Lorentz spaces, interpolation.

\footnotetext{
Abstract. In the paper we present three atomic decomposition theorems of Lorentz martingale spaces. With the help of atomic decomposition we obtain a sufficient condition for sublinear operator defined on Lorentz martingale spaces to be bounded. Using this sufficient condition, we investigate some inequalities on Lorentz martingale spaces. Finally we discuss the restricted weak-type interpolation, and prove the classical Marcinkiewicz interpolation theorem in the martingale setting.
}

\section{Introduction and Preliminaries}

The idea of atomic decomposition in martingale theory is derived from harmonic analysis. Just as it does in harmonic analysis, the method is key ingredient in dealing with many problems including martingale inequalities, duality, interpolation and so on, especially for small-index martingale and multi-parameter martingale. As well known, Weisz [8] 
gave some atomic decompositions on martingale Hardy spaces and proved many important theorems by atomic decompositions; Weisz [9] made a further study of atomic decompositions for weak Hardy spaces consisting of Vilenkin martingale, and proved a weak version of the Hardy-Littlewood inequality; Liu and Hou [5] investigated the atomic decompositions for vector-valued martingale and some geometry properties of Banach spaces were charactered; Hou and Ren [3] considered the vector-valued weak atomic decompositions and weak martingale inequalities; in [10], [11], the authors discussed the operator interpolation by atomic decompositions of weighted martingale Hardy spaces.

In this paper we present three atomic decomposition theorems for Lorentz martingale spaces $H_{p, q}^{s}, Q_{p, q}, D_{p, q}$. Applying these theorems, a sufficient condition for a sublinear operator defined on the Lorentz martingale spaces to be bounded is given. And then we obtain some continuous imbedding relationships among Lorentz martingale spaces. These are new versions of the basic inequalities in the classical martingale theory. Finally we also give a restricted weak-type interpolation theorem, and obtain the version of classical Marcinkiewicz interpolation theorem in the martingale setting.

Let $(\Omega, \Sigma, P)$ be complete probability space and $f$ be a measurable function defined on $\Omega$. The decreasing rearrangement of $f$ is the function $f^{*}$ defined by

$$
f^{*}(t)=\inf \{s>0: P(|f|>s) \leq t\} .
$$

We adopt the convention inf $\varnothing=\infty$. The Lorentz space $L_{p, q}(\Omega)=L_{p, q}, 0<$ $p<\infty, 0<q \leq \infty$, consists of those measurable functions $f$ with finite quasinorm $\|f\|_{p, q}$ given by

$$
\begin{aligned}
\|f\|_{p, q} & =\left(\frac{q}{p} \int_{0}^{\infty}\left[t^{1 / p} f^{*}(t)\right]^{q} \frac{d t}{t}\right)^{1 / q}, 0<q<\infty, \\
\|f\|_{p, \infty} & =\sup _{t>0} t^{1 / p} f^{*}(t), q=\infty .
\end{aligned}
$$

It will be convenient for us to use an equivalent definition of $\|f\|_{p, q}$, namely

$$
\begin{aligned}
\|f\|_{p, q} & =\left(q \int_{0}^{\infty}\left[t P(|f(x)|>t)^{1 / p}\right]^{q} \frac{d t}{t}\right)^{1 / q}, 0<q<\infty, \\
\|f\|_{p, \infty} & =\sup _{t>0} t P(|f(x)|>t)^{1 / p}, q=\infty .
\end{aligned}
$$

To check that these two expressions are the same, simply make the substitution $y=P(|f(x)|>t)$ and then integrate by parts.

It is well know that if $1<p<\infty$ and $1 \leq q \leq \infty$, or $p=q=1$, then $L_{p, q}$ is a Banach space, and $\|f\|_{p, q}$ is equivalent to a norm. However, for other 
values of $p$ and $q, L_{p, q}$ is only a quasi-Banach spaces. In particular,if $0<q \leq 1 \leq p$ or $0<q \leq p<1$ then $\|f\|_{p, q}$ is equivalent to a $q$ - norm. Recall also that a quasi-norm $\|\cdot\|$ in $X$ is equivalent to a $p$-norm, $0<p<1$, if there exists $c>0$ such that for any $x_{i} \in X, i=1, \ldots, n$

$$
\left\|x_{1}+\cdots+x_{n}\right\|^{p} \leq c\left(\left\|x_{1}\right\|^{p}+\cdots+\left\|x_{n}\right\|^{p}\right) .
$$

For all these properties, and more on Lorentz spaces, see for example [1, 2, 4]. Holder's inequality for Lorentz spaces is the following, which first appears in work of O'Neil [7],

$$
\|f g\|_{p, q} \leq c\|f\|_{p_{1}, q_{1}}\|g\|_{p_{2}, q_{2}}
$$

for all $0<p, q, p_{1}, q_{1}, p_{2}, q_{2} \leq \infty$ such that $\frac{1}{p}=\frac{1}{p_{1}}+\frac{1}{p_{2}}$ and $\frac{1}{q}=\frac{1}{q_{1}}+\frac{1}{q_{2}}$.

Let $\left\{\Sigma_{n}\right\}_{n \geq 0}$ a nondecreasing sequence of sub- $\sigma$-fields of $\Sigma$ such that $\Sigma=\bigvee \Sigma_{n}$. We denote the expectation operator and the conditional expectation operator relative to $\Sigma_{n}$ by $E$ and $E_{n}$, respectively. For a martingale $f=\left(f_{n}\right)_{n \geq 0}$, we define $\Delta_{n} f=f_{n}-f_{n-1}, n \geq 0$ (with convention $\left.f_{-1}=0, \Sigma_{-1}=\{\Omega, \Phi\}\right)$

$$
\begin{gathered}
M_{n}(f)=\sup _{0 \leq i \leq n}\left|f_{i}\right|, \quad M(f)=\sup _{n \geq 0}\left|f_{n}\right|, \\
S_{n}(f)=\left(\sum_{i=0}^{n}\left|\Delta_{i} f\right|^{2}\right)^{1 / 2}, \quad S(f)=\left(\sum_{n=0}^{\infty}\left|\Delta_{n} f\right|^{2}\right)^{1 / 2}, \\
s_{n}(f)=\left(\sum_{i=0}^{n} E_{i-1}\left|\Delta_{i} f\right|^{2}\right)^{1 / 2}, \quad s(f)=\left(\sum_{n=0}^{\infty} E_{n-1}\left|\Delta_{n} f\right|^{2}\right)^{1 / 2} .
\end{gathered}
$$

Denote by $\Lambda$, the set of all non-decreasing, non-negative and adapted r.v. sequences $\rho=\left(\rho_{n}\right)_{n \geq 0}$ with $\rho_{\infty}=\lim _{n \rightarrow \infty} \rho_{n}$. We shall say that a martingale $f=\left(f_{n}\right)_{n \geq 0}$ has predictable control in $L_{p, q}$ if there is a sequence $\rho=\left(\rho_{n}\right)_{n \geq 0} \in \Lambda$ such that

$$
\left|f_{n}\right| \leq \rho_{n-1}, \quad \rho_{\infty} \in L_{p, q} .
$$

As usually, we define Lorentz martingale spaces(see[1]),

$$
\begin{aligned}
H_{p, q}^{*} & =\left\{f=\left(f_{n}\right)_{n \geq 0}:\|f\|_{H_{p, q}^{*}}=\|M(f)\|_{p, q}<\infty\right\}, \\
H_{p, q}^{s} & =\left\{f=\left(f_{n}\right)_{n \geq 0}:\|f\|_{H_{p, q}^{s}}=\|s(f)\|_{p, q}<\infty\right\}, \\
H_{p, q}^{S} & =\left\{f=\left(f_{n}\right)_{n \geq 0}:\|f\|_{H_{p, q}^{S}}=\|S(f)\|_{p, q}<\infty\right\},
\end{aligned}
$$




$$
\begin{aligned}
Q_{p, q} & =\left\{f=\left(f_{n}\right)_{n \geq 0}: \exists\left(\rho_{n}\right)_{n \geq 0} \in \Lambda, \text { s.t. } S_{n}(f) \leq \rho_{n-1}, \rho_{\infty} \in L_{p, q}\right\} \\
\|f\|_{Q_{p, q}} & =\inf _{\rho}\left\|\rho_{\infty}\right\|_{p, q} \\
D_{p, q} & =\left\{f=\left(f_{n}\right)_{n \geq 0}: \exists\left(\rho_{n}\right)_{n \geq 0} \in \Lambda, \text { s.t. }\left|f_{n}\right| \leq \rho_{n-1}, \rho_{\infty} \in L_{p, q}\right\} \\
\|f\|_{D_{p, q}} & =\inf _{\rho}\left\|\rho_{\infty}\right\|_{p, q} .
\end{aligned}
$$

If we change the $L_{p, q}-$ norms in above definitions by $L_{p}-$ norms, we get the usual Hardy martingale spaces (see [6]).

Remark. The norms of $Q_{p, q}$ and $D_{p, q}$ are attainable respectively. For example, there exists $\left(\rho_{n}\right)_{n \geq 0} \in \Lambda, S_{n}(f) \leq \rho_{n-1}, \rho_{\infty} \in L_{p, q}$ such that $\|f\|_{Q_{p, q}}=\left\|\rho_{\infty}\right\|_{p, q}$, which is also called the optimal control.

It turns out that Lorentz spaces, as many other quasi-Banach spaces, admit some sort of atomic decomposition. Firstly we give the definition of an atom.

Definition [8]. A measurable function $a$ is called a $(1, p, \infty)$-atom (or $(2, p, \infty)$-atom or $(3, p, \infty)$-atom,respectively) if there exists a stopping time $\tau$ such that

(i) $a_{n}=E_{n} a=0, \quad n \leq \tau$,

(ii) $\|s(a)\|_{\infty} \leq P(\tau<\infty)^{-\frac{1}{p}}$ (or (ii) $\|S(a)\|_{\infty} \leq P(\tau<\infty)^{-\frac{1}{p}}$ or (ii) $\|M(a)\|_{\infty} \leq P(\tau<\infty)^{-\frac{1}{p}}$, respectively).

Throughout the paper, we denote the set of integers and the set of nonnegative integers by $Z$ and $N$, respectively. We write $A \preceq B$ if $A \leq c B$ for some positive constant $c$ independent of appropriate quantities involved in the expressions $A$ and $B$.

\section{Atomic decompositions}

Now we can present the atomic decompositions for Lorenz martingale spaces.

Theorem 2.1. If the martingale $f \in H_{p, q}^{s}, 0<p<\infty, 0<q \leq \infty$ then there exist a sequence $a^{k}$ of $(1, p, \infty)$-atoms and a positive real number sequence $\left(\mu_{k}\right) \in l_{q}$ such that

$$
f_{n}=\sum_{k \in Z} \mu_{k} a_{n}^{k}, n \in N
$$

and

$$
\left\|\left(\mu_{k}\right)_{k \in Z}\right\|_{l_{q}} \preceq\|f\|_{H_{p, q}^{s}} .
$$


Conversely, if $0<q \leq 1, q \leq p<\infty$, and the martingale $f$ has the above decomposition, then $f \in H_{p, q}^{s}$ and

$$
\|f\|_{H_{p, q}^{s}} \preceq \inf \left\|\left(\mu_{k}\right)_{k \in Z}\right\|_{l_{q}},
$$

where the inf is taken over all the preceding decompositions of $f$.

Proof. Assume that $f \in H_{p, q}^{s}, q \neq \infty$. Now consider the following stopping time for all $k \in Z$ :

$$
\tau_{k}=\inf \left\{n \in N: s_{n+1}(f)>2^{k}\right\}(\inf \phi=\infty) .
$$

The sequence of these stopping times is obviously non-decreasing. It easy to see that

$$
\begin{aligned}
\sum_{k \in Z}\left(f_{n}^{\tau_{k+1}}-f_{n}^{\tau_{k}}\right) & =\sum_{k \in Z}\left(\sum_{m=0}^{n} \chi_{\left\{m \leq \tau_{k+1}\right\}} \Delta_{m} f-\sum_{m=0}^{n} \chi_{\left\{m \leq \tau_{k}\right\}} \Delta_{m} f\right) \\
& =\sum_{k \in Z}\left(\sum_{m=0}^{n} \chi_{\left\{\tau_{k}<m \leq \tau_{k+1}\right\}} \Delta_{m} f\right)=f_{n} .
\end{aligned}
$$

Let $\mu_{k}=2^{k} 3 P\left(\tau_{k}<\infty\right)^{\frac{1}{p}}$, and

$$
a_{n}^{k}=\frac{f_{n}^{\tau_{k+1}}-f_{n}^{\tau_{k}}}{\mu_{k}} .
$$

If $\mu_{k}=0$ then we assume that $a_{n}^{k}=0$. Then for a fixed $k,\left(a_{n}^{k}\right)$ is a martingale. Since $s\left(f_{n}^{\tau_{k}}\right) \leq 2^{k}, s\left(f_{n}^{\tau_{k+1}}\right) \leq 2^{k+1}$,

$$
s\left(a_{n}^{k}\right) \leq \frac{s\left(f_{n}^{\tau_{k+1}}\right)+s\left(f_{n}^{\tau_{k}}\right)}{\mu_{k}} \leq P\left(\tau_{k}<\infty\right)^{-\frac{1}{p}}, n \in N,
$$

which implies that $\left(a_{n}^{k}\right)$ is a $L_{2}$-bounded martingale so that there exists $a^{k} \in L_{2}$ such that $E_{n} a^{k}=a_{n}^{k}$. If $n \leq \tau_{k}$ then $a_{n}^{k}=0$ and we get that $a^{k}$ is really a $(1, p, \infty)$ atom and

$$
\begin{aligned}
\left(\sum_{k \in Z}\left|\mu_{k}\right|^{q}\right)^{\frac{1}{q}} & =3\left(\sum_{k \in Z}\left(2^{k} P\left(\tau_{k}<\infty\right)^{\frac{1}{p}}\right)^{q}\right)^{\frac{1}{q}}=3\left(\sum_{k \in Z}\left(2^{k} P\left(s(f)>2^{k}\right)^{\frac{1}{p}}\right)^{q}\right)^{\frac{1}{q}} \\
& \preceq\left(\sum_{k \in Z} \int_{2^{k-1}}^{2^{k}} y^{q-1} d y P\left(s(f)>2^{k}\right)^{\frac{q}{p}}\right)^{\frac{1}{q}} \\
& \preceq\left(\sum_{k \in Z} \int_{2^{k-1}}^{2^{k}} y^{q-1} P(s(f)>y)^{\frac{q}{p}} d y\right)^{\frac{1}{q}} \\
& \preceq\left(\int_{0}^{\infty} y^{q-1} P(s(f)>y)^{\frac{q}{p}} d y\right)^{\frac{1}{q}} \preceq\|f\|_{H_{p, q}^{s}}
\end{aligned}
$$


For $q=\infty$, standard rectifications can be made.

Conversely, if $f$ has the above decomposition, then from $\left\|s\left(a^{k}\right)\right\|_{\infty} \leq$ $P\left(\tau_{k}<\infty\right)^{-\frac{1}{p}}$ and

$$
P\left(s\left(a^{k}\right)>y\right) \leq P\left(s\left(a^{k}\right) \neq 0\right) \leq P\left(\tau_{k}<\infty\right),
$$

we get

$$
\begin{aligned}
\left\|a^{k}\right\|_{H_{p, q}^{s}}^{q} & =q \int_{0}^{\infty} y^{q-1} P\left(s\left(a^{k}\right)>y\right)^{\frac{q}{p}} d y \\
& =q \int_{0}^{P\left(\tau_{k}<\infty\right)^{-\frac{1}{p}}} y^{q-1} P\left(s\left(a^{k}\right)>y\right)^{\frac{q}{p}} d y \\
& \leq P\left(\tau_{k}<\infty\right)^{\frac{q}{p}} \int_{0}^{P\left(\tau_{k}<\infty\right)^{-\frac{1}{p}}} y^{q-1} d y \leq \frac{1}{q} .
\end{aligned}
$$

For $0<q \leq 1, q \leq p<\infty,\|\cdot\|_{p, q}$ is equivalent to a $q-$ norm,

$$
\|f\|_{H_{p, q}^{s}}^{q} \leq\left\|\sum_{k \in Z} \mu_{k} s\left(a^{k}\right)\right\|_{p, q}^{q} \leq \sum_{k \in Z} \mu_{k}^{q}\left\|s\left(a^{k}\right)\right\|_{p, q}^{q} \preceq \sum_{k \in Z} \mu_{k}^{q},
$$

which gives the desired result.

Theorem 2.2. If the martingale $f \in Q_{p, q}, 0<p<\infty, 0<q \leq \infty$, then there exist a sequence $\left(a^{k}\right)$ of $(2, p, \infty)$ atoms and a real number sequence $\left(\mu_{k}\right) \in l_{q}$ such that

$$
f_{n}=\sum_{k \in Z} \mu_{k} a_{n}^{k}, \forall n \in N
$$

and

$$
\left(\sum_{k \in Z}\left|\mu_{k}\right|^{q}\right)^{\frac{1}{q}} \preceq\|f\|_{Q_{p, q}} .
$$

Conversely, if $0<q \leq 1, q \leq p<\infty$, and the martingale $f$ has the above decomposition, then $f \in Q_{p, q}$ and

$$
\|f\|_{Q_{p, q}} \preceq \inf \left(\sum_{k \in Z}\left|\mu_{k}\right|^{q}\right)^{\frac{1}{q}}
$$

where the inf is taken over all the above decompositions.

Proof. Suppose that $f \in Q_{p, q}$. Let $\beta=\left(\beta_{n}\right)_{n \geq 0}$ be the optimal control of $S_{n}(f)$, i.e., $\beta \in \Lambda, S_{n}(f) \leq \beta_{n-1},\|f\|_{Q_{p, q}}=\left\|\beta_{\infty}\right\|_{p, q}$. The stopping times $\tau_{k}$ are defined in this case by

$$
\tau_{k}=\inf \left\{n \in N: \beta^{n}>2^{k}\right\}(\inf \phi=\infty) .
$$


Let $a^{k}$ and $\mu_{k}(k \in Z)$ be defined as in the proof of Theorem 2.1. Then for a fixed $k,\left(a_{n}^{k}\right)$ is also a martingale. Since $S\left(f_{n}^{\tau_{k}}\right)=S_{\tau_{k}}(f) \leq \beta_{\tau_{k-1}} \leq$ $2^{k}, S\left(f_{n}^{\tau_{k+1}}\right) \leq 2^{k+1}$

$$
S\left(a_{n}^{k}\right) \leq \frac{S\left(f_{n}^{\tau_{k+1}}\right)+S\left(f_{n}^{\tau_{k}}\right)}{\mu_{k}} \leq P\left(\tau_{k}<\infty\right)^{-\frac{1}{p}}, n \in N .
$$

As in Theorem 2.1, we can show that $a^{k}$ is a $(2, p, \infty)$-atom. Also

$$
\begin{aligned}
\left(\sum_{k \in Z}\left|\mu_{k}\right|^{q}\right)^{\frac{1}{q}} & =3\left(\sum_{k \in Z}\left(2^{k} P\left(\tau_{k}<\infty\right)^{\frac{1}{p}}\right)^{q}\right)^{\frac{1}{q}} \\
& =3\left(\sum_{k \in Z}\left(2^{k} P\left(\beta_{\infty}>2^{k}\right)^{\frac{1}{p}}\right)^{q}\right)^{\frac{1}{q}} \\
& \preceq\left\|\beta_{\infty}\right\|_{p, q}=\|f\|_{Q_{p, q}} .
\end{aligned}
$$

Conversely, if $a^{k}$ is $(2, p, \infty)$-atom, one can show that $\left\|a^{k}\right\|_{H_{p, q}^{S}}^{q} \leq \frac{1}{q}$. The rest can be proved similar to Theorem 2.1.

Theorem 2.3. If the martingale $f \in D_{p, q}, 0<p<\infty, 0<q \leq \infty$, then there exist a sequence $\left(a^{k}\right)$ of $(3, p, \infty)$-atoms and a real number sequence $\left(\mu_{k}\right) \in l_{q}$ such that

$$
f_{n}=\sum_{k \in Z} \mu_{k} a_{n}^{k}, n \in N
$$

and

$$
\left(\sum_{k \in Z}\left|\mu_{k}\right|^{q}\right)^{\frac{1}{q}} \preceq\|f\|_{D_{p, q}} .
$$

Conversely, if $0<q \leq 1, q \leq p<\infty$, and the martingale $f$ has the above decomposition, then $f \in D_{p, q}$ and

$$
\|f\|_{D_{p, q}} \preceq \inf \left(\sum_{k \in Z}\left|\mu_{k}\right|^{q}\right)^{\frac{1}{q}}
$$

where the inf is taken over all the above decompositions.

The proof of Theorem 2.3 is similar to that of Theorem 2.2 and so we omit it.

\section{Bounded operators on Lorentz martingale spaces}

As one of the applications of the atomic decompositions, we shall obtain a sufficient condition for a sublinear operator to be bounded from Lorentz martingale spaces to function Lorentz spaces. Applying the condition to 
$M f, S f$ and $s f$, we deduce a series of inequalities on Lorentz martingale spaces.

An operator $T: X \rightarrow Y$ is called a sublinear operator if it satisfies

$$
|T(f+g)| \leq|T f|+|T g|,|T(\alpha f)| \leq|\alpha||T f|,
$$

where $X$ is a martingale space, $Y$ is a measurable function space.

Theorem 3.1. Let $T: H_{r}^{s} \rightarrow L_{r}$ be a bounded sublinear operator for some $1 \leq r<\infty$. If

$$
P(|T a|>0) \preceq P(\tau<\infty)
$$

for all $(1, p, \infty)$-atoms a, where $\tau$ is the stopping time associated with a, then for $0<p<r, 0<q \leq \infty$, we have

$$
\|T f\|_{p, q} \leq\|f\|_{H_{p, q}^{s}}, \quad f \in H_{p, q}^{s} .
$$

Proof. Assume that $f \in H_{p, q}^{s}$. By Theorem 2.1, $f$ can be decomposed into the sum of a sequence of $(1, p, \infty)$-atoms. For any fixed $y>0$ choose $j \in Z$ such that $2^{j-1} \leq y<2^{j}$ and let

$$
f=\sum_{k \in Z} \mu_{k} a^{k}=\sum_{k=-\infty}^{j-1} \mu_{k} a^{k}+\sum_{k=j}^{\infty} \mu_{k} a^{k}=: g+h .
$$

Recall that $\mu_{k}=2^{k} 3 P^{1 / p}\left(\tau_{k}<\infty\right)$ and $s\left(a^{k}\right)=0$ on the set $\left\{\tau_{k}=\infty\right\}$. we have

$$
\begin{aligned}
\|g\|_{H_{r}^{s}} & \leq\left(\int_{\Omega}\left(\sum_{k=-\infty}^{j-1} \mu_{k} s\left(a^{k}\right)\right)^{r} d P\right)^{1 / r} \leq \sum_{k=-\infty}^{j-1} \mu_{k}\left(\int_{\Omega}\left(s\left(a^{k}\right)\right)^{r} d P\right)^{1 / r} \\
& \leq \sum_{k=-\infty}^{j-1} \mu_{k}\left(\int_{\left\{\tau_{k} \leq \infty\right\}}\left\|s\left(a^{k}\right)\right\|_{\infty}^{r} d P\right)^{1 / r} \\
& \leq \sum_{k=-\infty}^{j-1} \mu_{k} P\left(\tau_{k}<\infty\right)^{-\frac{1}{p}} P\left(\tau_{k}<\infty\right)^{\frac{1}{r}} \\
& =\sum_{k=-\infty}^{j-1} 2^{k} P\left(\tau_{k}<\infty\right)^{\frac{1}{r}} \\
& =\sum_{k=-\infty}^{j-1} 2^{k} P\left(s(f)>2^{k}\right)^{\frac{1}{r}}
\end{aligned}
$$


It follows from the boundedness of $T$ that

$$
\begin{aligned}
P(|T g|>y) & \leq y^{-r} E|T g|^{r} \preceq y^{-r}\|g\|_{H_{r}^{s}}^{r} \\
& \preceq y^{-r}\left(\sum_{k=-\infty}^{j-1} 2^{k} P\left(s(f)>2^{k}\right)^{\frac{1}{r}}\right)^{r} \\
& =y^{-r}\left(\sum_{k=-\infty}^{j-1} 2^{k\left(1-\frac{p}{r}\right)} 2^{k \frac{p}{r}} P\left(s(f)>2^{k}\right)^{\frac{1}{r}}\right)^{r} \\
& \preceq y^{-r}\left(\sum_{k=-\infty}^{j-1} 2^{k\left(1-\frac{p}{r}\right)}\right)^{r}\|s f\|_{p, \infty}^{p} \\
& \leq y^{-p}\|s f\|_{p, \infty}^{p} .
\end{aligned}
$$

On the other hand, since $|T h| \leq \sum_{k=j}^{\infty} \mu_{k}\left|T a^{k}\right|$, we get

$$
\begin{aligned}
P(|T h|>y) & \leq P(|T h|>0) \leq \sum_{k=j}^{\infty} P\left(\left|T a^{k}\right|>0\right) \\
& =\sum_{k=j}^{\infty} P\left(\tau_{k}<\infty\right)=\sum_{k=j}^{\infty} 2^{-k p} 2^{k p} P\left(s f>2^{k}\right) \\
& \leq \sum_{k=j}^{\infty} 2^{-k p}\|s f\|_{p, \infty}^{p} \\
& \preceq y^{-p}\|s f\|_{p, \infty}^{p} .
\end{aligned}
$$

Since $T$ is subliear,

$$
P(|T f|>y) \leq P\left(|T g|>\frac{y}{2}\right)+P\left(|T h|>\frac{y}{2}\right) \preceq y^{-p}\|s f\|_{p, \infty}^{p},
$$

and thus for all $0<p<r, T: H_{p, \infty}^{s} \rightarrow L_{p, \infty}$ is bounded. Now for any fixed $0<p<r$, we can choose $0<p_{0}, p_{1}<r, 0<\theta<1$ satisfying $\frac{1}{p}=\frac{1-\theta}{p_{0}}+\frac{\theta}{p_{1}}$. From interpolation theorem (see Theorem 5.11 [2]) and the boundedness of sublinear is hereditary for the interpolation spaces, we obtain for $0<q \leq \infty$

$$
T: H_{p, q}^{s}=\left(H_{p_{0}, \infty}^{s}, H_{p_{1}, \infty}^{s}\right)_{\theta, q} \rightarrow\left(L_{p_{0}, \infty}, L_{p_{1}, \infty}\right)_{\theta, q}=L_{p, q}
$$

is bounded. Hence

$$
\|T f\|_{p, q} \preceq\|f\|_{H_{p, q}^{s}} .
$$

On the lines of the proof of Theorem 3.1, we can prove the following Theorems 3.2 and 3.3 by using Theorems 2.2 and 2.3 , respectively. 
Theorem 3.2. Let $T: Q_{r} \rightarrow L_{r}$ be a bounded sublinear operator for some $1 \leq r<\infty$. If

$$
P(|T a|>0) \preceq P(\tau<\infty)
$$

for all $(2, p, \infty)$-atoms $a$, where $\tau$ is the stopping time associated with $a$, then for $0<p<r, 0<q \leq \infty$, we have

$$
\|T f\|_{p, q} \preceq\|f\|_{Q_{p, q}}, \quad f \in Q_{p, q} .
$$

Theorem 3.3. Let $T: D_{r} \rightarrow L_{r}$ be a bounded sublinear operator for some $1 \leq r<\infty$. If

$$
P(|T a|>0) \preceq P(\tau<\infty)
$$

for all $(3, p, \infty)$-atoms $a$, where $\tau$ is the stopping time associated with a, then for $0<p<r, 0<q \leq \infty$, we have

$$
\|T f\|_{p, q} \preceq\|f\|_{D_{p, q}}, \quad f \in D_{p, q} .
$$

Theorem 3.4. For all martingale $f=\left(f_{n}\right)_{n \geq 0}$ the following imbeddings hold:

1) For $0<p<2,0<q \leq \infty$,

$$
H_{p, q}^{s} \hookrightarrow H_{p, q}^{*}, \quad H_{p, q}^{s} \hookrightarrow H_{p, q}^{S},
$$

for $p>2,0<q \leq \infty$,

$$
H_{p, q}^{*} \hookrightarrow H_{p, q}^{s}, \quad H_{p, q}^{S} \hookrightarrow H_{p, q}^{s} .
$$

2) For $0<p<\infty, 0<q \leq \infty$,

$$
\begin{array}{cc}
Q_{p, q} \hookrightarrow H_{p, q}^{*}, \quad Q_{p, q} \hookrightarrow H_{p, q}^{S}, \quad Q_{p, q} \hookrightarrow H_{p, q}^{s} \\
D_{p, q} \hookrightarrow H_{p, q}^{*}, \quad D_{p, q} \hookrightarrow H_{p, q}^{S}, \quad D_{p, q} \hookrightarrow H_{p, q}^{s} .
\end{array}
$$

Proof. 1) The maximal operator $T f=M f$ is sublinear, and $\|M f\|_{2} \leq\|s f\|_{2}$. If $a$ is any $(1, p, \infty)$-atom and $\tau$ is the corresponding stopping time, then $\{|T a|>0\}=\{|M a|>0\} \subset\{\tau<\infty\}$ and hence $P(|T a|>0) \leq P(\tau<\infty)$. It follows from Theorem 3.1 that

$$
\|M f\|_{p, q} \preceq\|f\|_{H_{p, q}^{s}}, \quad(0<p<2) .
$$

Similarly, consider the operator $T f=S f$. We get $\|S f\|_{p, q} \preceq\|f\|_{H_{p, q}^{s}}$. Conversely, we use vector-valued interpolation (see [6]) to obtain the case $p>2,0<q \leq \infty$.

In fact, we can regard martingale spaces as the subspaces of sequence spaces. Consider the operator $Q: L_{p}\left(l_{\infty}\right) \rightarrow L_{p}$ defined by $Q\left(f_{n}\right)=s(f)=$ 
$\left(\sum_{n=0}^{\infty} E_{n-1}\left|\Delta_{n} f\right|^{2}\right)^{1 / 2}$. For $p \geq 2$, we know that $\|s(f)\|_{p} \preceq\|M(f)\|_{p}=$ $\left\|\sup _{n \geq 0}\left|f_{n}\right|\right\|_{p}$ and so $Q: L_{p}\left(l_{\infty}\right) \rightarrow L_{p}$ is bounded for all $p \geq 2$. For any fixed $p>2$, we can choose $p_{0}, p_{1}>2,0<\theta<1$ satisfying $\frac{1}{p}=\frac{1-\theta}{p_{0}}+\frac{\theta}{p_{1}}$. Consequently $Q: L_{p_{i}}\left(l_{\infty}\right) \rightarrow L_{p_{i}}$ is bounded, $i=0,1$. By interpolation, for $0<q \leq \infty$,

$$
Q: L_{p, q}\left(l_{\infty}\right)=\left(L_{p_{0}}\left(l_{\infty}\right), L_{p_{1}}\left(l_{\infty}\right)\right)_{\theta, q} \rightarrow\left(L_{p_{0}}, L_{p_{1}}\right)_{\theta, q}=L_{p, q}
$$

is bounded. Hence we obtain

$$
\|s(f)\|_{p, q} \preceq\left\|\sup _{n \geq 0}\left|f_{n}\right|\right\|_{p, q}=\|M(f)\|_{p, q},
$$

which gives $H_{p, q}^{*} \hookrightarrow H_{p, q}^{s}$. By considering $Q$ defined on the sequence space $L_{p}\left(l_{2}\right)$, we can similarly prove $H_{p, q}^{S} \hookrightarrow H_{p, q}^{s}$

2) For all $0<r<\infty,\|M(f)\|_{r},\|S(f)\|_{r},\|s(f)\|_{r} \preceq\|f\|_{Q_{r}}$ and $\|M(f)\|_{r},\|S(f)\|_{r},\|s(f)\|_{r} \preceq\|f\|_{D_{r}}$. Note that $a_{n}^{k}=0$ on the set $\left\{n \leq \tau_{k}\right\}$.

Thus

$$
\chi\left(n \leq \tau_{k}\right) E_{n-1}\left|\Delta_{n} a^{k}\right|^{2}=E_{n-1} \chi\left(n \leq \tau_{k}\right)\left|\Delta_{n} a^{k}\right|^{2}=0 .
$$

Hence $s\left(a^{k}\right)=0$ on the set $\left\{\tau_{k}=\infty\right\}$. By Theorem 3.2 and 3.3, the assertion is proved.

Remark. If we put $p=q$ in the above embeddings, Theorem 2.11 in [8] can be deduced.

Remark. We conjecture that for $1 \leq p<\infty, 0<q \leq \infty, H_{p, q}^{S}=H_{p, q}^{*}$, however our method doesn't show it.

\section{Restricted weak type interpolation}

We say that a sublinear operator $T$ is of Lorentz- $s$ restricted weak-type $(p, q)$ if $T$ maps $H_{p, 1}^{s}$ to $L_{p, \infty}$. For convenience, we call $T$ as restricted weak-type $(p, q)$. Then we have the next interpolation from one restricted weak-type estimate to another.

Theorem 4.1. Let $T$ be of restricted weak-type $\left(p_{i}, q_{i}\right)$ for $i=0,1$, and $1<p_{i}, q_{i}<\infty$. Put

$$
\frac{1}{p}=\frac{1-\theta}{p_{0}}+\frac{\theta}{p_{1}}, \frac{1}{q}=\frac{1-\theta}{q_{0}}+\frac{\theta}{q_{1}}, \quad \forall 0 \leq \theta \leq 1 .
$$

Then $T$ is also of restricted weak-type $(p, q)$.

Proof. Suppose that $f \in H_{p, 1}^{s}$. From Theorem 2.1, $f=\sum_{k \in Z} \mu_{k} a^{k}, a^{k}$ is a $(1, p, \infty)$-atom with respect to the stopping time $\tau_{k}$, and $\sum_{k \in Z}\left|\mu_{k}\right| \preceq$ 
$\|f\|_{H_{p, 1}^{s}}$. Now we can estimate $\left\|T a^{k}\right\|_{q, \infty} \preceq 1$. In fact

$$
\begin{aligned}
\left\|T a^{k}\right\|_{q, \infty} & =\sup _{t>0} t^{\frac{1}{p}}\left(T a^{k}\right)^{*}(t)=\sup _{t>0}\left(t^{\frac{1}{q_{0}}}\left(T a^{k}\right)^{*}(t)\right)^{1-\theta}\left(t^{\frac{1}{q_{1}}}\left(T a^{k}\right)^{*}(t)\right)^{\theta} \\
& \leq\left\|T a^{k}\right\|_{q_{0}, \infty}^{1-\theta}\left\|T a^{k}\right\|_{q_{1}, \infty}^{\theta} \\
& \preceq\left\|s a^{k}\right\|_{p_{0}, 1}^{1-\theta}\left\|s a^{k}\right\|_{p_{1}, 1}^{\theta} \\
& \leq\left\|s a^{k}\right\|_{2 p_{0}, 2 p_{0}}^{1-\theta}\left\|\chi_{\left\{\tau_{k}<\infty\right\}}\right\|_{2 p_{0}, l}^{1-\theta}\left\|s a^{k}\right\|_{2 p_{1}, 2 p_{1}}^{\theta}\left\|\chi_{\left\{\tau_{k}<\infty\right\}}\right\|_{2 p_{1}, m}^{\theta} \\
& \leq P\left(\tau_{k}<\infty\right)^{-\frac{1}{p}}\left(P\left(\tau_{k}<\infty\right)^{\frac{1-\theta}{2 p_{0}}} P\left(\tau_{k}<\infty\right)^{\frac{\theta}{2 p_{1}}}\right)^{2} \\
& \leq 1
\end{aligned}
$$

where $l=\frac{2 p_{0}}{2 p_{0}-1}$ and $m=\frac{2 p_{1}}{2 p_{1}-1}$. Consequently,

$$
\|T f\|_{q, \infty} \leq \sum_{k \in Z}\left|\mu_{k}\right|\left\|T a^{k}\right\|_{q, \infty} \preceq \sum_{k \in Z}\left|\mu_{k}\right| \preceq\|f\|_{H_{p, 1}^{s}}
$$

and the proof is complete.

Now we show how restricted weak-type estimate can be transferred to strong type. It is also the version of the classical Marcinkiewicz interpolation theorem in the martingale setting(see Theorem 4.13 in [1]).

Theorem 4.2. Let $T$ be of restricted weak-type $\left(p_{i}, q_{i}\right)$ for $i=0,1$, and $1<p_{i}<\infty, 1<q_{i} \leq \infty, q_{0} \neq q_{1}$. Put

$$
\frac{1}{p}=\frac{1-\theta}{p_{0}}+\frac{\theta}{p_{1}}, \frac{1}{q}=\frac{1-\theta}{q_{0}}+\frac{\theta}{q_{1}}, \quad 0 \leq \theta \leq 1 .
$$

Then $T$ is of type $\left(H_{p, r}^{s}, L_{q, r}\right)$, for $0<r<1$ and $r \leq q$.

Proof. For $0<r<1$ and $r \leq q$, we know that $\|\cdot\|_{q, r}$ is equivalent to a $r$-norm, so it is enough to prove $\|T a\|_{q, r} \preceq 1$, for all $(1, p, \infty)$-atoms. Once it is proved then from Theorem 2.1,

$$
\|T f\|_{q, r}^{r} \leq \sum_{k \in Z} \mu_{k}^{r}\|T a\|_{q, r}^{r} \preceq \sum_{k \in Z} \mu_{k}^{r} \preceq\|f\|_{H_{p, r}^{s}}^{r} .
$$

Now we shall show $\|T a\|_{q, r} \preceq 1$. Consider the case $q_{1}, q_{2}<\infty$. From the proof of Theorem 4.1, it is easy to see that

$$
\|a\|_{H_{p_{i}, 1}^{s}}^{p_{i}} \leq P(\tau<\infty)^{1-\frac{p_{i}}{p}}, \quad i=0,1
$$


Thus, for $q_{0}<q<q_{1}$, we get

$$
\begin{aligned}
\frac{1}{q}\|T a\|_{q, r}^{q} & =\int_{0}^{\infty} y^{r-1} P(|T a|>y)^{\frac{r}{q}} d y \\
& \leq \int_{0}^{\delta} y^{r-1}\left(\frac{1}{y}\|a\|_{H_{p_{0}, 1}^{s}}\right)^{\frac{q_{0} r}{q}} d y+\int_{\delta}^{\infty} y^{r-1}\left(\frac{1}{y}\|a\|_{H_{p_{1}, 1}^{s}}\right)^{\frac{q_{1} r}{q}} d y \\
& \preceq \delta^{\frac{r}{q}\left(q-q_{0}\right)} P(\tau<\infty)^{\frac{r q}{q}\left(\frac{1}{p_{0}}-\frac{1}{p}\right)}+\delta^{\frac{r}{q}\left(q-q_{1}\right)} P(\tau<\infty)^{\frac{r q_{1}}{q}\left(\frac{1}{p_{1}}-\frac{1}{p}\right)}
\end{aligned}
$$

Take $\delta=P(\tau<\infty)^{\alpha}$ with $\alpha$ satisfying

$$
q \alpha=\left(\frac{1}{p_{0}}-\frac{1}{p}\right) /\left(\frac{1}{q}-\frac{1}{q_{0}}\right)=\left(\frac{1}{p_{1}}-\frac{1}{p}\right) /\left(\frac{1}{q}-\frac{1}{q_{1}}\right)
$$

In fact, from $\frac{1}{p}=\frac{1-\theta}{p_{0}}+\frac{\theta}{p_{1}}, \frac{1}{q}=\frac{1-\theta}{q_{0}}+\frac{\theta}{q_{1}}$ we find that $q \alpha=\left(\frac{1}{p_{0}}-\frac{1}{p_{1}}\right) /\left(\frac{1}{q_{1}}-\right.$ $\left.\frac{1}{q_{0}}\right)$, and

$$
\frac{r}{q}\left[\alpha\left(q-q_{0}\right)+q_{0}\left(\frac{1}{p_{0}}-\frac{1}{p}\right)\right]=\frac{r}{q}\left[\alpha\left(q-q_{1}\right)+q_{1}\left(\frac{1}{p_{1}}-\frac{1}{p}\right)\right]=0 .
$$

Then $\|T a\|_{q, r}^{q} \preceq 1$.

When one of $q_{i}$ is $\infty$, say $q_{1}=\infty$, the proof is modified. More precisely, we have

$$
\|T a\|_{\infty} \preceq\|a\|_{H_{p_{1}, 1}^{s}} \preceq P(\tau<\infty)^{\frac{1}{p_{1}}-\frac{1}{p}} .
$$

Thus, from $\frac{1}{p}=\frac{1-\theta}{p_{0}}+\frac{\theta}{p_{1}}, \frac{1}{q}=\frac{1-\theta}{q_{0}}$

$$
\begin{aligned}
\frac{1}{q}\|T a\|_{q, r}^{q} & =\int_{0}^{\|T a\|_{\infty}} y^{r-1} P(|T a|>y)^{\frac{r}{q}} d y \\
& \leq \int_{0}^{\|T a\|_{\infty}} y^{r-1}\left(\frac{1}{y}\|a\|_{H_{p_{0}, 1}^{s}}\right)^{\frac{q_{0} r}{q}} d y \\
& \preceq P(\tau<\infty)^{\frac{r q_{0}}{q}\left(\frac{1}{p_{0}}-\frac{1}{p}\right)} P(\tau<\infty)^{\frac{r}{q}\left(q-q_{0}\right)\left(\frac{1}{p_{1}}-\frac{1}{p}\right)} \\
& \preceq 1
\end{aligned}
$$

the assertion follows.

Remark. From Theorems 2.2 and 2.3, we can conclude that the familiar results hold for $Q_{p, 1}$ and $D_{p, 1}$. We shall not state those explicitly.

Acknowledgement. The project was supported by the National Natural Science Foundation of China (10671147) and China Scholarship Council (2007U13085). The authors would like to thank the referee and the editor for their helpful suggestions. 


\section{References}

[1] C. Bennett and R. Sharply, Interpolation of Operator, Academic press, 1988.

[2] J. Bergh, J. Lofstrom, Interpolation Spaces, an Introduction, Berlin, Heidelberg, New York, Springer, 1976.

[3] Y.L. Hou and Y.B. Ren, Vector-valued weak martingale Hardy spaces and atomic decompositions, Acta Math. Hungarica, 115 (2007), 235246.

[4] R. Hunt, On L(p,q) spaces, L'Ens. Math. 12 (1966), 249-275.

[5] P.D. Liu and Y.L. Hou, Atomic decomposition of Banach-space-valued martingales, Sci in China, Serices A Math. 42 (1999), 38-47.

[6] R. L. Long, Martingale Spaces and Inequalities, Beijing, Peking Univ Press, 1993.

[7] R. O'Neil, Convolution operator and $L(p, q)$ spaces, Duke. Math. J., 30 (1963), 129-143.

[8] F. Weisz, Martingale Hardy Spaces and Their Applications in Fourier Analysis, Lecture Notes in Math, Vol. 1568, New York:Sping-verlag, 1994.

[9] F. Weisz, Bounded operator on weak Hardy spaces and applications, Acta Math. Hungarica, 80 (1998), 249-264.

[10] J. Yong, F. Li Ping and L. Peide Interpolation on weighted Lorentz martingale spaces, Sci in China, Serices A Math., 50 (2007), 12171226 .

[11] J. Yong, P.D. Liu and L.H. Peng, Interpolation for martingale Hardy spaces over weightedmeasure spaces, Acta Math. Hungar., 120 (2008), 127-139.

Jiao Yong and Peng Lihua

School of Mathematical Science and Computing Technology

Central South University, Chang Sha 410081

China

(E-mail : jjiao@univ-fcomte.fr)

Liu Peide

School of Mathematics and Statistics

Wuhan University

Wuhan 430072

China

(Received : November 2007) 


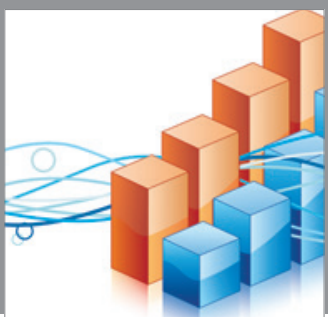

Advances in

Operations Research

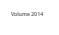

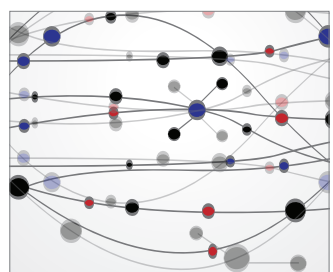

\section{The Scientific} World Journal
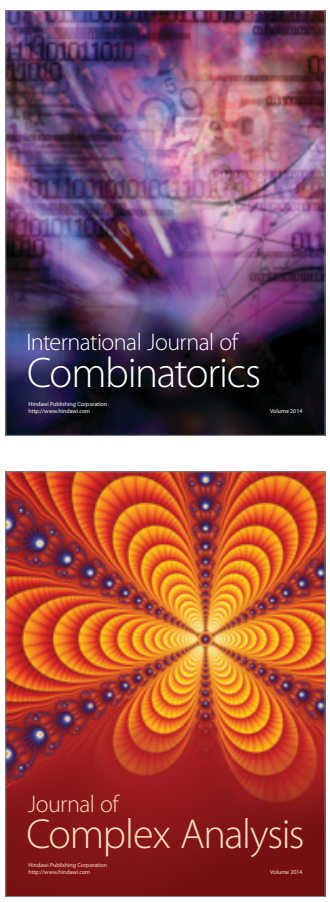

International Journal of

Mathematics and

Mathematical

Sciences
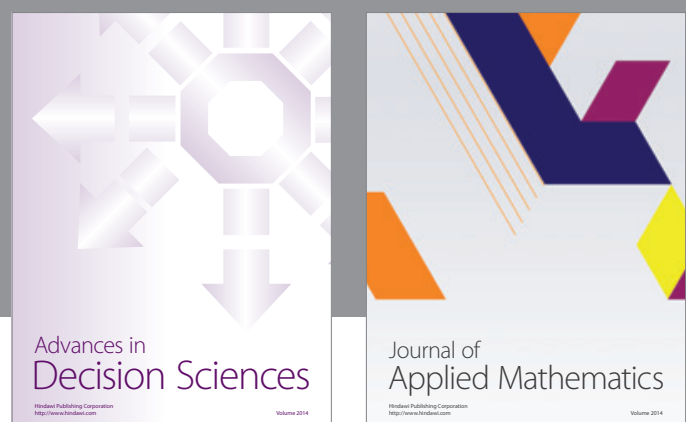

Journal of

Applied Mathematics
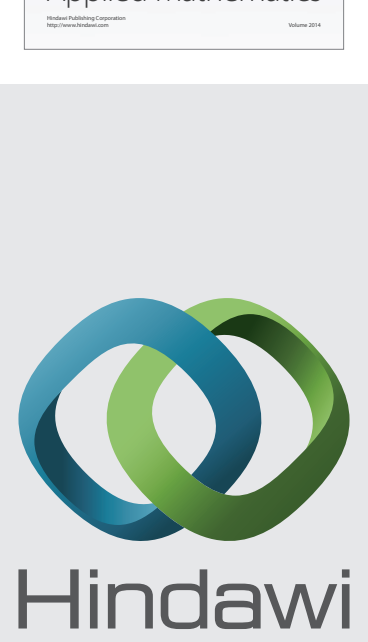

Submit your manuscripts at http://www.hindawi.com
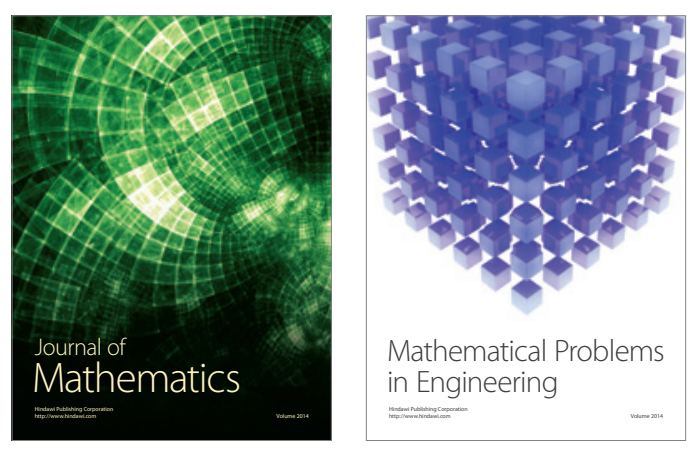

Mathematical Problems in Engineering
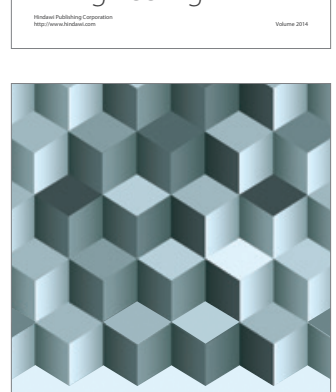

Journal of

Function Spaces
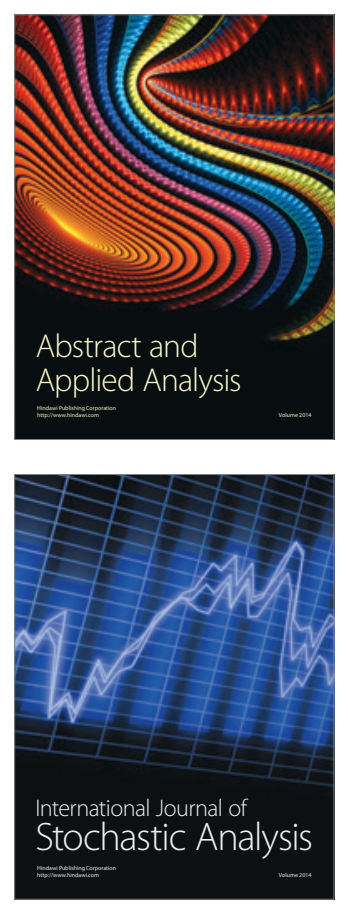

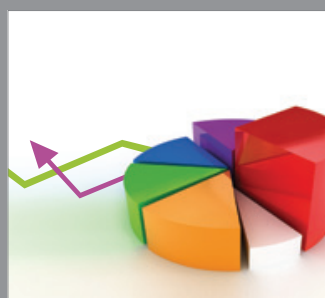

ournal of

Probability and Statistics

Promensencen
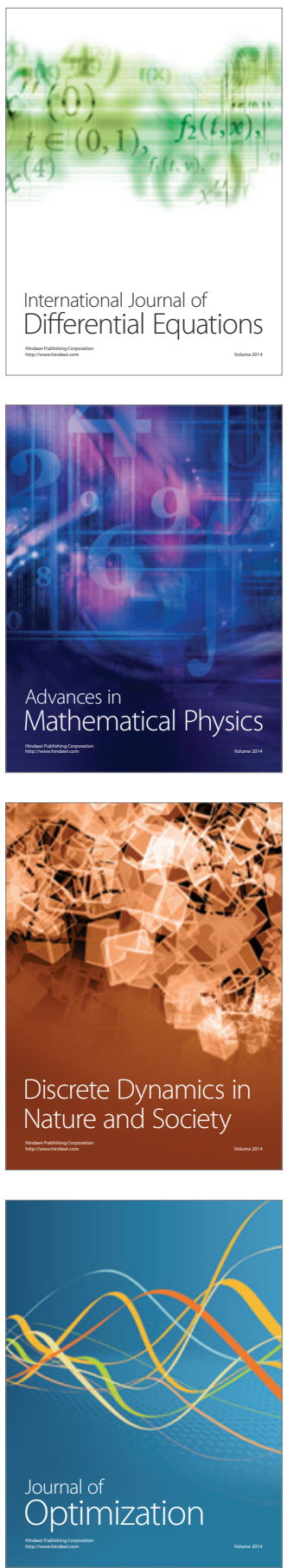